\title{
Image Compression Study using Diversified Approaches
}

\author{
${ }^{1}$ Jayshree R. Pansare, ${ }^{2}$ Ketki R. Jadhav \\ ${ }^{1,2}$ Department of Computer Engineering, M.E.S. College of Engineering, Pune, India \\ jayshree.pansare23@gmail.com; ketkijadhav174@gmail.com
}

\begin{abstract}
Image compression plays an important role in minimizing irrelevance and redundancy of digital images for efficient transmission and storage. It saves large storage capacity and transmission bandwidth. The aim of image compression algorithm is to reduce amount of data required to represent image with less degradation without loss. This paper studies diversified approaches for compression of images such as FELICS, Wavelet transformation based approach, Prediction based approach, Combination of method based approach and Block coding based approach etc. Under different approaches there are different techniques for compression of image and gives better results than previous state-of-art techniques. Different methods gives different compression ratio, saves memory, and gives high performance. They have their own strength and weaknesses.
\end{abstract}

Keywords: Image compression; FELICS; Block coding; DCT-DKT HWT; LAR-LLC; DDCPM; APC; CALIC.

\section{Introduction}

In today's world, ever growing field of digital technology comes with tremendous changes in daily life. Most of us want image representation of content rather than text for better understanding and visual appearance. It is required that we reduce total number of bits for image compression. Image compression is divided into two categories, lossless compression and lossy compression. In lossless compression, original image reconstructed without loss of any information. While lossy compression, irrelevant information discarded to achieve benefits of larger storage space. Compression is achieved using reduction of three essential information redundancies: 1) Coding redundancy, which is available when not exactly ideal code words are utilized. (2) Interpixel redundancy, which results from correlations between the pixels of an image. (3) Psychovisual redundancy, which is because of information that is disregarded by the human visual framework. The paper represents different approaches for compression of images.

We divided different methods into different sections according to their approaches. The paper fragmented the methods in five approaches: FELICS based approach, Wavelet transformation based approach, Prediction based approach, Block coding based approach, Combination of methods based approach. FELICS based approach consist of original FELICS and its extension. FELICS gives speed which is five times faster than JPEG [2]. VLSI-oriented FELICS algorithm method based on parallelism mechanism improves compression ratio by 35 per on average of original FELICS [20]. Wavelet transformation based approaches mainly based on transformation of RGB frames into different co. channels, So they can be used for compression. Wavelet transformation based approach consist of prediction error based method, Hierarchical Prediction and Context Adaptive Coding implemented algorithm, Combination of vector quantization and HWT method, Color space transformation method, LAR-LLC method. 
Jayshree R. Pansare, Ketki R. Jadhav; Image Compression Study using Diversified Approaches. Advances in Image and Video Processing, Volume 5 No 1, February (2017); pp: 13-19

Prediction error based method is much better than other wavelet based techniques such as WDR. JPEG2000 and SPIHT and gives better compression performance for different size images [11]. Hierarchical Prediction and Context Adaptive Coding implemented algorithm improves 7.10per and 18.89per coding gain over JPEG-2000 and JPEG-XR respectively [17]. Combination of vector quantization and HWT gives acceptable image quality with 32per compression ratio on an average [19]. Color space transformation method uses two transformation methods RDgDb and LDgEb gives better results for 8 natural photographic and artificial images, set of 24 photographic image given by the Kodak corporation, 10 high resolution images[10]. LAR-LLC uses HD-ST transform for improving performance of LAR [18]. Prediction based approaches consist of super-Spatial Structure Prediction, Bayesian Predictor Combination, Two-Stage Prediction. Super-spatial prediction method used for high-frequency image structure component encoding, such as edge, patterns and textures. Bayesian

Predictor Combination method used for improvement the performance of APC. Proposed APC-MAP scheme generates smaller bit rates than APC. Two-Stage Prediction method performs two-stage prediction on hyperspectral images using BPS and data correlation [12][16][14]. Combination methods consist of fusion of LZW and BCH algorithm method and fusion of DDPCM and Golomb-Rice algorithm [7][15]. Block based approach consist of geometric-primitives-based compression scheme, ParameterAssistant Inpainting scheme, Two-Stage Modeling Method [3][9][13].

\section{The Existing Techniques In Image Compression}

\subsection{FELICS Based Approach}

In this section, we emphasize on various approaches related to FELICS as follows:

\subsubsection{FELICS (Fast and Efficient Lossless Compression System method)}

Method uses system that runs very fast and gives efficient lossless compression. It uses raster-scan and uses a pixel of two nearest neighbors to obtain an approximate probability distribution for its intensity. And uses the prediction and error modeling. For coding it uses Golomb or Rice codes, adjusted binary codes and single bits [2].

\subsubsection{The VLSI-oriented, fast, efficient, lossless image compression system (FELICS) algorithm}

The VLSI-oriented fast, efficient, lossless image compression system (FELICS) algorithm is combination of simplified adjusted binary code and Golomb-Rice code with storage-less parameter selection. In simplified adjusted binary code, adjusted binary code arithmetic operations get reduced gives improved processing speed. The storage-less k parameter selection reduces the data dependency for Golomb-Rice code. In comparison with original FELICS algorithm, the Compression ratio is improved about 35per on average. Improve coding efficiency with simple arithmetic operation. Experiment results shows that the proposed method presents superior performance in parallelism-efficiency and power efficiency compared with other existing works. The maximum throughput can achieve 4.36 $\mathrm{Gb} / \mathrm{s}$. Regarding high definition (HD) display applications, our encoding capability can achieve a highquality specification of full-HD 1080p at $60 \mathrm{~Hz}$ with complete red, green, blue color components[20].

\subsection{Wavelet Transformation Based Approach}

In this section, we focus on various approaches related to Wavelet Transformation as follows:

\subsubsection{Prediction error based compression of color images using WDR coding}

This method is combination of wavelet difference reduction and prediction error quantization. This method reduces correlation between RGB planes using RGB to $\mathrm{YCbCr}$ transform and generates no of 
sub-images using decomposition of each transformed plane. Compression of sub-images of $Y$ happens independently while compression of each of sub-images of $\mathrm{Cb}$ and $\mathrm{Cr}$ planes is done through combination of quantization, WDR and Huffman coding techniques. The experimental results and analysis of the proposed work demonstrate its superiority both in terms of objective and subjective fidelity criteria. When this method is applied on different size images like Airplane, pepper, Lena each of size $512 * 512$ and Girl, Couple, House and Zelda each of size $256 * 256$ it gives better PSNR and bpp values. This compression method achieves high compression performance and attempt equivalent image quality when image is reconstructed. The proposed scheme outperforms over wavelet based compression techniques (SPIHT and WDR) with very large extent and its compression performance is even better to the state of art JPEG2000. It also saves large storage space [11].

\subsubsection{Hierarchical Prediction and Context Adaptive Coding for Lossless Color Image Compression}

This paper presents a new lossless color image compression algorithm. This paper designs an edge directed predictor and context adaptive model for hierarchical scheme. The method uses lower, upper and left sided pixels for prediction of pixel to be encoded. For lossless image compression, RGB is first transformed into $\mathrm{YCbCr}$ by $\mathrm{RCT}$. $\mathrm{Y}$ channels are encoded by a conventional grayscale image compression algorithm. The prediction of chrominance channel is done through hierarchical predication scheme. The hierarchical scheme decomposes chrominance image into two subimages: a set of even numbered rows Xe and a set of odd numbered rows Xo respectively. After the encoding Xe the even row subimage, method uses pixels in Xe for prediction of pixel in odd row subimage. Proposed method gives higher coding gain than JPEG2000 and JEPG-XR in many cases. The proposed method and several conventional methods have been tested on the Kodak image set, some medical images, and digital camera images, and it is shown that average bit rate reductions over JPEG2000 for these sets are shown to be 7.10per, 13.55per, and 5.52per respectively [17].

\subsubsection{Color Image Compression using Vector Quantization and Hybrid Wavelet Transform}

The combination of Hybrid wavelet transforms and vector quantization has been used for increasing compression ratio. Proposed method combines two different transforms Discrete Kekre Transform (DKT) and Discrete Cosine Transform (DCT) are used to get DKT-DCT HWT. Then applies VQ technique on transformed image to generate 16 and 32 size codebook. The paper studies different $V Q$ algorithms and their performance. Application of vector quantization increases compression ratio. The results of algorithms shows that KMCG and KFCG gives faster execution and beat performance of LBG algorithm KFCG combined with hybrid wavelet transform gives lowest distortion and acceptable image quality at compression ratio 192. As compared to LBG, KMCG gives less error at compression ratio 64 and KFCG gives least error among all with better image quality. Both KMCG and KFCG are faster in execution and give good quality image and compression [19].

\subsubsection{New simple and efficient color space transformations for lossless image Compression}

The method implements two new transformations RDgDb and LDgEb. The proposed transformations are compared with existing RCT, YCoCg-R and optimal KLT for 3 set of test images and for different compression algorithms such as JPEG-LS, JPEG2000 and JPEG XR. Experimental results shows that RDgDb gives best ratios for JPEGXR and JPEG2000, while for a specific set or in case of JPEG-LS its compression ratios are either the best or within $0.1 \mathrm{bpp}$ from the best. The variant of RDgDb, modularRDgDb gives overall best ratios with JPEG-LS. Also another transformation LDgDb gives better complexity and ratios than RCT and YCoCg-R, but worse than RDgDb[10]. 


\subsubsection{LAR-LLC: A Low-Complexity Multiresolution Lossless Image Codec}

To achieve, better low-complexity solution paper presents a new scalable locally adaptive resolution lossless, low-complexity (LAR-LLC) image codec. The process of modification of LAR framework goes through different stages. In the first step, the hierarchical diagonal S transforms (HD-ST) is proposed for new reversible transform. Prediction of transforming coefficients is done in a second stage by considering both inter- and intra-level information. On the final stage, classification and Huffman coding has been applied. Classification is done through context modeling approach. LAR-LLC saves the decoding latency by 75.94per, which is higher than 65.85per of JPEGXR, and a little less than LPEEG which has 82.01 per. The proposed LAR-LLC has a speed gain up to 76.11per compared with JPEG2K, and this result is better than LJPEG 2.35per and JPEGXR [18].

\subsection{Prediction Based Approach}

In this section, we highlight on various approaches related to prediction as follows:

\subsubsection{Lossless Image Compression Using Super-Spatial Structure Prediction}

Super-spatial structure prediction scheme develop an efficient image compression scheme based on super-spatial prediction of structural units. It finds an optimal prediction of structure components within previously encoded image regions. It divides the image in two types of regions: structure regions and non structure regions. Super-spatial prediction encodes structure regions, while NSR can be encoded with conventional image compression methods such as CALIC. When compared to VQbased image encoders, it has the flexibility to incorporate multiple H. 264-style prediction modes. When compared to other neighborhood-based prediction methods, such as GAP and H.264 Intra prediction, it allows the block to find the best match from the whole image which significantly reduces the prediction residual by up to 79per[12].

\subsubsection{Bayesian Predictor Combination for Lossless Image Compression}

Adaptive predication combination (APC) combines multiple predictors for lossless image compression and act as center for state-of-art algorithms. In this paper, a Bayesian parameter estimation scheme is proposed. The proposed method improves predication performance of APC. APC uses two parameters mean and variance for each predictor for error distribution. The proposed method assumes mean parameter of APC for each predictor as non zero, rather than zero as used in APC for error distribution and makes a maximum likelihood estimate for the two parameters. Then applies Bayesian approach. The proposed algorithm APC-MAP has good prediction performance than APC and APC-ML. However, it has small computational overhead than APC and APC-ML. It is better than APC for any number of fixed predictors. APC-MAP requires fewer predictors compared to APC, and achieves a comparable running time [16].

\subsubsection{An Efficient Lossless Compression Scheme for Hyperspectral Images Using Two-Stage Prediction}

This method presents an efficient lossless compression scheme for hyperspectral images. The proposed scheme based two stage predictor. The two stage predictor consists of intra-/inter-band prediction and Backward Pixel Search (BPS). The median predictor used in JPEG-LS was employed here because of its simplicity. The stage-2 predictor takes prediction results from stage-1 predictor as an initial value and conducts a Backward Pixel Search (BPS) scheme of the current band for the final value. Experimental results show that given two-stage prediction scheme performs better than all other schemes for Airborne Visible/Infrared Imaging Spectrometer (AVIRIS) 1997 images. Show that the BPS 
scheme, aimed at exploiting calibration-induced data correlation, is effective on Airborne Visible/Infrared Imaging Spectrometer (AVIRIS) 1997 images where such artifacts are significant. The proposed work outperforms all other schemes under comparison in this category. For the newer Consultative Committee for Space Data Systems images where calibration-induced artifacts are minimized, the stage-1 predictor alone achieves better compression performance [14].

\subsection{Combination Method Based Approach}

We focus on different combination methods as follows:

\subsubsection{Lossless Image Compression Technique Using Combination Methods}

This method is combination of LZW and $\mathrm{BCH}$ algorithms. This approach works as follows: first, it performs the well-known Lempel-Ziv-Welch algorithm on the image. What comes out of the first step is forward to the second step where the Bose, Chaudhuri and Hocquenghem error correction and detected algorithm is used. To improve the compression ratio, the proposed approach applies the $\mathrm{BCH}$ algorithms repeatedly until inflation is detected [7].

\subsubsection{A Lossless Color Image Compression Architecture Using a Parallel Golomb-Rice Hardware CODEC}

The proposed method is a combination of differential-differential pulse coded Modulation (DDPCM) and Golomb-Rice coding. It goes through two stages: 1) Application of DDPCM on an original image frame which is arranged as $m$ by $n$ sub-window arrays. The output of this step is one main piece called as seed and $m^{*} n-1$ pieces of differential data. 2) Output of $1^{\text {st }}$ step goes to 2 nd step which uses Golomb-Rice algorithm to encode differential data for generation of lossless compressed data. The main piece seed is used in its uncompressed form. This method is mainly applied to benchmark images. The proposed method can guarantee a high compression rate and throughput to carry out real-time lossless CODEC operation. Minimize both memory requirement and bandwidth is proposed. On different sub-window arrays the scheme gives a different compression ratio. As sub-window array size increases, compression ratio also increases. Proposed method shows better results in terms of hardware complexity, performance, and power consumption. The usability of the proposed scheme would be limited due to its lower compression ratio [15].

\subsection{Block Coding Based Approach}

This section present multiple approaches based on block coding as follows:

\subsubsection{A Geometric-Primitives-Based Compression Scheme For Testing Systems-On-A-Chip}

Geometric Primitive-based compression scheme for testing system uses novel lossless compression method for testing system-on-a-chip based on geometric contours. This method increases test data size to make test data reduction imperative. The system increases compression ratio. Test data is divided into blocks and then block encoded separately. System fails when test data size is too large [3].

\subsubsection{Block-Based Image Compression with Parameter-Assistant In painting}

This method consists of model class for representing different distribution of image regions. Based on that, an input image on the encoder side is divided into featured and non-featured regions at block level. The featured blocks same as image regions of predefined model class are coded by a few parameters, whereas the non-featured blocks are coded using existing DCT-based methods. At the decoder side, the featured regions are restored through PAI based on both delivered parameters and 
Jayshree R. Pansare, Ketki R. Jadhav; Image Compression Study using Diversified Approaches. Advances in Image and Video Processing, Volume 5 No 1, February (2017); pp: 13-19

surrounding information. Experimental results show that our method better than JPEG in featured regions by an average bit-rate saving of 76per at similar perceptual quality levels. PAI gives much less artifacts than JPEG, especially in the sky of the Pole, the wall of the Statue and the Sunglow of Kodim20 [9].

\subsubsection{A Two-Stage Modeling Method for Compressing Binary Images by Arithmetic Coding}

This method is combination of block coding and arithmetic coding. This reduces use of arithmetic coding because of his slow operation property. It uses QM-coder is highly sophisticated arithmetic coder, which will be the point of comparison made in this paper later on. The block coding is used only in the modeling, so arithmetic coding remains an autonomous phase. So it is possible to use different versions of arithmetic coding. This method is best for binary images such as black and white images mostly better for more white images [13].

\section{Conclusion}

There is lot of techniques for image compression. We studies different methods and divide them in five different approaches such as FELICS based approach, Wavelet transformation based approach, Prediction based approach, Combination of methods based approach, Block coding based approach.

According to similarity between different methods we divide the methods in different approaches as above. The methods have their own advantages and disadvantages. Given methods gives better performance than there state-of-art methods such as JPEG2000, JPEG2K, JPEGXR, APC etc. A different method uses different parameters to calculate performance of system, such as compression ratio, speed, bandwidth, reconstructed image quality, used memory space etc. The future work includes the implementation of system which is better than these methods and gives better performance than these methods.

\section{ACKNOWLEDGMENT}

It gives me great pleasure and satisfaction in presenting seminar on "Review on Existing Techniques for image compression," I would like to thank my project guide, Prof. J. R. Panasare mam for her guidance and support. Without her constant support, guidance and assistance this seminar report would not have been completed. Without their coordination, guidance and reviewing this task could not be completed alone. I would like to thank all those, who have directly or indirectly helped me for the completion of the work during this project.

\section{REFERENCES}

[1]. J. Zobel and A. Moffat, "Adding compression to a full-text retrieval system," Softw.-Pract. Exper, vol. 25, no. 8, pp. 891-903, 1995.

[2]. P. Franti, "A fast and efficient compression method for binary images," Signal Process., Image Commun., vol. 6, no. 1, pp. 69-76, Mar. 1994.

[3]. P. Franti and O. Nevalainen, "A two-stage modeling method for compressing binary images by arithmetic coding," Comput. J., vol. 36, no. 7, pp. 615-622, 1993.

[4]. X. Wu and N. Memon, "Context-based, adaptive, lossless image coding," IEEE Trans. Commun., vol. 45, no. 4, pp. 437-444, Apr. 1997. 
Advances in Image and Video Processing Volume 5, Issue 1, February, 2017

[5]. A. Podlasov and P. Franti, "Lossless image compression via bit-plane separation and multilayer context tree modeling,"J. Electron. Imag, vol. 15, no. 4, pp. 043-009, Nov. 2006.

[6]. P. Franti, E. Ageenko, P. Kopylov, S. Grhn, and F. Berger, "Compression of map images for real-time applications," Image Vis. Comput., vol. 22, no. 13, pp. 1105-1115, 2004.

[7]. A. Alarabeyyat, S. Al-Hashemi, T. Khdou1, M. Hjouj Btoush, S. Bani-Ahmad, R. Al-Hashemi, "Lossless Image Compression Technique Using Combination Methods," Journal of Software Engineering and Applications, pp 752-763, 2012.

[8]. A. Bookstein and S. T. Klein, "Is Huffman coding dead?"Computing, vol. 50, no. 4, pp. 279-296, 1993.

[9]. A. El-Maleh, S. al Zahir, and E. Khan, "A geometric-primitives-based compression scheme for testing systems-on-a-chip," in Proc. 19th IEEE Comput. Soc. Conf. VLSI Test Symp., pp. 54-59, Apr. 2001.

[10]. Roman Starosolski, "New simple and efficient color space transformations for lossless image compression," J. Vis. Commun. Image R. vol. 25, pp. 1056-1063, 2014.

[11]. Manoj Kumar, Ankita Vaish, "Prediction error based compression of color images using WDR coding," Int. J. Electron. Commun. (AE), vol. 70, pp. 1164-1171, 2016.

[12]. Xiwen OwenZhao, Zhihai HenryHe, "Lossless Image Compression Using Super-Spatial Structure Prediction,” IEEE Trans. Signal Process, vol. 17, no. 4, pp. 383-386, April 2010.

[13]. Zhiwei Xiong, Xiaoyan Sun, FengWu, "Block-Based Image Compression With Parameter-Assistant Inpainting," IEEE Trans. Image Process, vol. 19, NO. 6, pp. 1651-1657, June 2010.

[14]. Cheng-Chen Lin and Yin-Tsung Hwang, "An Efficient Lossless Compression Scheme for Hyperspectral Images Using Two-Stage Prediction," IEEE Trans. Geoscience and remote sensing, vol. 7, no. 3, pp. 558-562, July 2010.

[15]. Hong-Sik Kim, Joohong Lee, Hyunjin Kim, Sungho Kang, and Woo Chan Park, "A Lossless Color Image Compression Architecture Using a Parallel Golomb-Rice Hardware CODEC,"IEEE Trans. Circuits Syst. For Video Technol., vol. 21, no. 11, pp. 1581-1587, November 2011.

[16]. Andrew Martchenko and Guang Deng, "Bayesian Predictor Combination for Lossless Image Compression, "IEEE Trans. Image Process., vol. 22, no. 12, pp. 5263-5269, December 2013.

[17]. Seyun Kim and Nam Ik Cho, "Hierarchical Prediction and Context Adaptive Coding for Lossless Color Image Compression," IEEE Trans. Image Process, vol. 23, no. 1, pp. 445-448, January 2014.

[18]. Yi Liu, Olivier Dforges, and Khouloud Samrouth ,"LAR-LLC: A Low-Complexity Multiresolution Lossless Image Codec," IEEE Trans. Circuits Syst. for Video Technol., vol. 26, no. 8, pp. 1490-1501, august 2016.

[19]. H. B. Kekrea, Prachi Natub and Tanuja Sarodec, "Color Image Compression using Vector Quantization and Hybrid Wavelet Transform," Procedia Computer Science, vol. 89, pp. 778 784, 2016.

[20]. Tsung-Han Tsai, Yu-Hsuan Lee, and Yu-Yu Lee, "Design and Analysis of High-Throughput Lossless Image Compression Engine Using VLSI Oriented FELICS Algorithm," IEEE trans. on very large scale integr.(vlsi) syst., vol. 18, no. 1, pp. 39-52, January 2010. 